\title{
Auditory system: development, genetics, function, aging, and diseases
}

\author{
Bernd Fritzsch $^{1} \cdot$ Marlies Knipper $^{2} \cdot$ Eckhard Friauf $^{3}$
}

Published online: 9 June 2015

(C) Springer-Verlag Berlin Heidelberg 2015

The auditory system is, like no other sensory system, tied into the human social evolution as the basic information highway for language and thus the evolution of an information flow-centered society. The evolution of an auditory system enabled the evolution of language and, through the unlimited assignment of meaning to spoken words, including the semantic and emotional content, the selection of abstract information processing as a basis of modern human societies. Our modern society has transited into a novel area where predominantly visual information through hand-held devices will be as important as the communication via spoken word. But even this new development will increasingly rely on the spoken word and thus the auditory system for communication purposes now that functional devices exist that can convert spoken words into written text.

The impact of the auditory system on our daily lives goes far beyond this historical reflection and its modern, computerbased incarnation. Anybody suffering from hearing loss can easily relate to the difficulty one faces when communication through spoken words becomes problematic, for instance as we age. The unpleasant and depressing feeling of sitting in a

Bernd Fritzsch

bernd-fritzsch@uiowa.edu

Marlies Knipper

marlies.knipper@uni-tuebingen.de

Eckhard Friauf

eckhard.friauf@biologie.uni-kl.de

1 Department of Biology, University of Iowa, CLAS, 143 BB, Iowa City 52242, IA, USA

2 Hearing Research Centre Tübingen, University of Tübingen, Elfriede Aulhornstr. 5, 72076 Tübingen, Germany

3 Animal Physiology Group, Department of Biology, University of Kaiserslautern, Post Box 3049, 67653 Kaiserslautern, Germany group of people talking to each other with a hearing-impaired person sitting in between, unable to extract meaning from the multitude of information reaching the brain, is an increasing problem as we move toward super-aged societies to which people above 65 years will contribute $25 \%$ or more. This 'silver tsunami' will be a defining feature of our societies for the foreseeable future, necessitating deep reflections on how to move forward by retaining those suffering from variable degree of hearing impairment as viable members of our societies.

Understanding both the impact of hearing for communication and the age-related inability to do so requires a detailed comprehension of the development, function, and functional decline of the hearing system to establish guiding principles as to how to help those suffering from communication inabilities through congenital defects or experiencing the gradual loss of once well-established abilities to extract meaning out of sound. Embedded in this is the problem of hearing non-existing or 'phantom sound', usually referred to as tinnitus. Such 'sound without source' can further compromise already declining abilities to extract meaning out of existing sound by masking or distracting. To achieve such a basic insight to ultimately cure these well-known and emerging problems in hearing requires detailing issues of overall development of the auditory system, functional development of features such as directional and frequency-specific hearing, and processing of complex sounds and encoding neuronal information, as well as understanding the pathology and the age-related functional decline of the auditory system. This special issue comprises 24 contributions highlighting specific aspects related to this broad perspective. It is our hope that the reader will find the insights provided by these papers helpful in a better understanding of the auditory system and its importance for various areas of neurobiology as well as development and aging in the nervous system.

The 24 topics are combined into three major parts describing mainly (1) Structural and functional differentiation 
phenomena during early development (chapters 1-7), (2) Crucial aspects of sound processing in the healthy auditory system (chapters 8-14), and (3) Age- and disease-related changes in the auditory system (chapters 15-24). Below, we provide a snapshot of the coverage by each of the 24 chapters of this special issue.

1. The introductory chapter by Fritzsch et al. (2014) sets the stage. The review, entitled "Inner ear development: Building a spiral ganglion and an organ of Corti out of unspecified ectoderm", highlights critical steps in ear development. These steps transform an otic placode, the ectodermal anlage of the developing ear, into the highly specialized patterning of the organ of Corti. The organ of Corti transforms sound into electrical signals, thus providing input to the brain to process sound-related information. The review also addresses the unique innervation of the organ of Corti by two types of afferents and efferents reaching distinct types of inner and outer hair cells. Finally, the chapter provides a summary of major steps and the molecular guidance of these steps by a handful of wellcharacterized transcription factors to generate a functional organ of Corti.

2. The chapter by Sienknecht (2015), entitled "Current concepts of hair cell differentiation and planar cell polarity in inner ear sensory organs", focuses on the development of the highly polarized hair cell bundles (i.e., stereocilia) and the molecular machinery guiding this planar cell polarity process which is so crucial for the function of the sensory epitheliae. The chapter provides an overview of the aspects that are currently becoming clear but also identifies areas that are less clear in terms of their molecular basis. Such issues need to be known in order to guide a functional restoration of the organ of Corti that has lost hair cells due to age or various diseases.

3. The molecular basis of auditory system development is further expanded in the chapter "The emerging framework of mammalian auditory hindbrain development" by Nothwang et al. (2015). This chapter highlights the molecular basis of brainstem development with special emphasis on the origin of auditory hindbrain nuclei in distinct embryonic compartments of the brainstem, the rhombomeres. These compartments are defined by a partially overlapping Hoxgene code that provides identity to specific rhombomeres. This chapter also offers an overview on guidance molecules for the auditory circuit assembly and the role played by activity in fine tuning the initially formed connections. Combined, these data deliver a framework that helps to establish clues to the causes and consequences of hearing loss-related disorders.

4. The chapter by Durruthy-Durruthy and Heller (2014) provides conceptually novel insights into the sequence of gene activation to highlight "Applications for single cell trajectory analysis in inner ear development and regeneration". Using computer algorithms, the authors extract information from existing PCR data on developing mouse neuroblast lineage. Importantly, the authors use three different approaches and exemplify the strengths and weaknesses of each approach. The authors stress that this information is essential to guide attempts towards restoring lost neurosensory cells in the organ of Corti, a widely accepted beneficial target of hearing restoration .

5. The review by Seymour and Pereira (2015), entitled "Survival of auditory hair cells", takes a closer look at the molecular basis of hair cell viability, with the aim of rescuing hair cells from various damages achieved by loud sound, ototoxic drugs, and certain anti-neoplastic drugs known to cause progressive and permanent hearing impairment accompanied by hair cell loss. The review introduces novel approaches using antioxidants, anti-inflammatory drugs and apoptosis inhibitors to enhance hair cell survival. Whereas some of the advances are promising, the chapter argues that conceptual issues around protection versus rescue need to be taken into account to move the field forward.

6. The chapter by Wang and Bergles (2014), "Spontaneous activity in the developing auditory system", stresses the role of spontaneous activity in refining the tonotopic map laid down by the processes introduced in the three preceding chapters. Molecular developmental events organize the organ of Corti such that a tonotopic map becomes established through local differentiation of stiffness and hair cells along its length, including the innervation via afferents and efferents. The connectivity of the organ of Corti with the cochlear nuclei is discussed, which is achieved by the afferent auditory nerve fibers prior to any electrical activity. Spontaneous activity in the pre-hearing period helps to further finetune these connections. Where these spontaneous activities arise is an ongoing debate. Both inner hair cells and their supporting cells, possibly through the release of ATP, are discussed. This article details how the activity-mediated process is involved in the maturation of the nascent circuits of the auditory system.

7. When the cells in the organ of Corti approach maturation during early development and have progressed with fine-tuning their proper wiring with the neurons in the cochlear nuclear complex under the influence of spontaneous $\mathrm{Ca}^{2+}$ action potentials, maturation of the pre-and post-synaptic elements between inner hair cells and auditory nerve fibers marks another critical step in accomplishing mature sound processing. This crucial aspect of maturation is summarized in the chapter by Knipper et al. (2015) with the title "Specific 
synaptopathies diversify brain responses and hearing disorders. You lose the gain from early life...". There are various feedback control systems for normal development of the pre- and post-synaptic structures. These feedback loops need to be understood in the context of diseases linked to inner hair cell synaptopathies and correlated differential disturbance of different auditory fiber types that may lead to disturbances of adaptive central responsiveness. In this context, Knipper et al. particularly stress the relationships of these early processes to agedependent hearing loss, tinnitus, and hyperacusis.

8. Information flow via the synapses between inner hair cells and the many type I afferents (spiral ganglion neurons) requires a detailed understanding of the synaptic mechanisms working at these unusual ribbon synapses. The chapter by Wichmann and Moser (2015), entitled "Relating structure and function on inner hair cell ribbon synapses", provides a detailed overview on this functionally unique assembly that is so crucial for the high fidelity of information transfer in the peripheral auditory system. Attention is given to important conceptual aspects of the molecular release machinery that allows stimulus-secretion coupling, docking, modes of endocytosis, and vesicle reformation. Recent work providing unique insights into this essential process is reviewed and put into the context of hair cell communication via the conspicuous ribbon synapse.

9. The functional differences of spiral ganglion cells along the cochlear turns that transmit various frequencies from the organ of Corti to the brain are reviewed by Davis and Crozier (2015) in the chapter "Dynamic firing properties of type I spiral ganglion neurons". Data suggest that neuronal firing properties defy the apparent morphological uniformity and suggest that postnatal exposure to various neurotrophins regulates properties related to the tonotopic position of a given spiral ganglion neuron. It is suggested that the different physiological properties are an essential feature that needs to develop properly to ensure the flow of auditory information into the brain.

10. The timing and the rates of action potentials in the spiral ganglion neurons comprise the neuronal code of virtually all acoustic information transmitted from the organ of Corti to the brain. In their chapter "Basic response properties of auditory nerve fibers: a review", Heil and Peterson (2015) provide a summary of some of the basic response properties of type I afferents and what little we know about type II afferents. The authors address issues like spontaneous activity, frequency tuning curves, rate-level functions, spike-rate adaptation and recovery as well as phase locking. They also address the effects of acoustic trauma on some of these properties. Aging, congenital disorders, infections, exposure to excessively loud sound, and treatment with ototoxic drugs are causes of sensorineural hearing loss. In the presence of such hearing loss, response properties of auditory nerve fibers change manifold. Some changes are profound, others are only subtle and may last for variable periods, potentially compounding each during a life span.

11. Aiming to better understand neuronal sound processing in the auditory system, Rudnicki and Hemmert (2015) in their review "Modelling auditory coding: from sound to spikes" summarize the strengths and pitfalls of current cochlear model organisms. The critical evaluation of available models in their review provides a most suitable tool that allows researchers to find their best model to answer their specific research question and also provides guidance that allow researchers to switch between the animal models to achieve their research goal.

12. Depending on the recent history of pre-synaptic activity, post-synaptic responses increase or decrease in size. The activity-dependent changes in synaptic strength are called synaptic facilitation and synaptic depression, respectively. Synaptic plasticity is well analyzed in the auditory system, particularly because of the presence of giant pre-synaptic terminals, i.e., endbulbs and calyces of Held, which, because of their size, are accessible for patch-clamp and imaging experiments. The chapter by Friauf et al. (2015) entitled "Synaptic plasticity in the auditory system: a review" discusses a total of 14 variants of plasticity in the mammalian and avian auditory brainstem, stretching from short-term depression (STD) to long-term potentiation. Of particular interest is a metaanalysis, in which the authors compare STD between auditory and non-auditory synapses. From this analysis, it follows that most auditory synapses display less STD than non-auditory ones, enabling reliable, failure-free transmission of synaptic signals even at action potential rates exceeding $100 \mathrm{~Hz}$. Surprisingly, the calyx of Held synapses deviate from this rule, being less resistant to depression while displaying a frequency at halfmaximal depression as low as $1.4 \mathrm{~Hz}$.

13. Stimulus-specific adaptation (SSA) is a process during which neuronal responses specifically adapt to repeated sounds without generalization to other sounds. SSA occurs in the inferior colliculus, the medial geniculate body, and the auditory cortex. The phenomenon of SSA is thought to underlie mechanisms of novelty detection and recognition of acoustic objects. The current knowledge of what is behind SSA and its relation to the remarkable capability of the auditory system to rapidly detect novel or deviant stimuli is summarized in the chapter "Deviance detection in auditory subcortical structures: what can we learn from their neurochemistry and neural connectivity?" by Duque et al. (2015). It is argued that proper development of temporal features 
of sound processing define adaptive or non-adaptive central response behavior as a consequence of the development of the special capability of auditory neurons to show SSA. Indeed, it is not clear whether SSA depends on proper temporal signatures of auditory fiber signaling or is part of a central integration step. SSA is a crucial capability of animals, which may be critical for their survival, as their life may depend on detecting unexpected stimuli above background.

14. To improve auditory signaling, the brain makes use of multisensory integration. Several neurons in the auditory system, e.g., fusiform cells in the dorsal cochlear nucleus, are not exclusively 'auditory' but integrate auditory and somatosensory information. In the chapter entitled "Listening to another sense: somatosensory integration in the auditory system", Wu et al. (2014) address the very appealing issue of multisensory integration and explore the process of combining information from multiple sensory modalities. They do so by focusing on anatomical, physiological, functional, and pathological aspects of this integration. As multisensory integration occurs throughout the auditory system, convergence of auditory and sensory inputs is discussed at each ascending auditory station, from the cochlear nuclei to the auditory cortex. The review highlights the intricacy of sensory processing and offers a multisensory perspective regarding the understanding of sensory disorders.

15. The brain has the remarkable capability to respond in an adaptive or non-adaptive way to sensory deprivation. Processing new sound information may depend on the sound experiences we encounter during the critical period after the onset of hearing. This becomes most evident when brain structures are analyzed that are deprived of hearing during these earliest stages of sound processing. Ryugo (2014) summarizes in his review "Auditory neuroplasticity, hearing loss, and cochlear implants" the profound structural deficits that congenitally deaf white cats experience upon hearing loss. It also describes to what extent these structural deficits can be restored through electrical stimulation mediated by cochlear implants.

16. While "Asymmetric and unilateral hearing losses in children" have traditionally been underappreciated, we are now beginning to understand their effect on development and the underlying pathophysiologic mechanisms. The objective of this review by Vila and Lieu (2015) is to illustrate to the non-otolaryngologist the importance of asymmetric and unilateral hearing loss in children, how to detect it, what causes it, the resulting consequences on the child's development, and how it can be addressed once diagnosed. In addition, they present additional problems requiring future research in this emerging field.
17. Hearing loss within a critical early time period after hearing onset obviously also shapes part of the behavioral responses, such as the vocal behavior. This is demonstrated in the congenitally deaf white cat by Hubka et al. (2014) in their chapter "Auditory feedback modulates development of kitten vocalizations". Evidence is provided that not all aspects of a given stimulus, but only characteristic aspects of the structure of the vocalizations, critically depend on timely auditory feedback that appears to influence neuronal motor control early in life.

18. Valuable insights into the role of experience in shaping perception can be obtained by studying the effects of blindness or other forms of sensory deprivation on the intact auditory sense. In the chapter "Crossmodal plasticity and hearing capabilities following blindness", King (2015) explores how blind individuals in particularly depend on their hearing. There is extensive evidence that they can develop superior auditory skills, either as a result of plasticity within the auditory system or through the recruitment of functionally relevant occipital cortical areas that lack their normal visual inputs. Because spatial processing normally relies on close interactions between vision and hearing, much of the research in this area has focused on the effects of blindness on auditory localization. Although enhanced auditory spatial skills have been reported in many studies, some aspects of spatial hearing are impaired in the absence of vision. In this case, the effects of crossmodal plasticity may reflect a balance between adaptive changes that compensate for blindness and the role vision normally plays, particularly during development, in calibrating the brain's representation of auditory space.

19. Details of the efferent feedback loop that act on cochlear activity regulated by the olivo-cochlear bundle are summarized in the review by Nouvian et al. (2015) with the topic "Cochlear efferents in developing adult and pathological conditions". The detailed overview summarizes the crucial modulating and protecting role of the efferent feedback from the olivo-cochlear bundle on outer and inner hair cells, how this feedback develops, and how it influences hearing during pathological conditions.

20. Proper development of afferent fiber characteristics under the control of top-down influence from the brain can be derailed to lead to unusual processing of sound. The review of Eggermont and Roberts (2014), "Tinnitus: animal models and findings in humans", provides a comprehensive overview about the relationship of altered auditory nerve fiber input through deafferentation and altered central processing, with an emphasis on changes in the auditory cortex. In particular, the pitfalls of correlations of observations between animals and 
patients are illustrated to relate limits of model data transfer to human situations.

21. Needless to say, comprehension of complex acoustic signals, such as speech, requires not only proper function of the periphery but also of the central auditory system. Aging with its various physiological and behavioral changes is associated with degenerative processes in all organ systems. Age-related hearing impairment (presbycusis), the number one communication disorder and one of the three most frequent chronic medical conditions of the elderly, can involve both peripheral changes (loss of hair cells) and changes in the central auditory system, such as the composition of neurotransmitters and receptors. Ouda et al. (2015) tackle this issue in the chapter "Age-related changes in the auditory system". The authors review our contemporary knowledge about age-related changes in the central auditory system of animals (mostly aging mice, rats, and monkeys) as well as humans. They summarize compelling evidence that age-related hearing loss has two components, one that represents deterioration of the auditory periphery and the other, central presbycusis, which may lead to deficits in central auditory processing, for example a reduction in gap detection.

22. A characteristic age-dependent modification in the central auditory system is the focus of the chapter of Williamson et al. (2014): "Auditory brainstem cap responses start to decline in middle age mice: a novel physiological biomarker for age-related hearing loss". The main findings that unravel delayed peak latency and decreased peak amplitudes in various mice strains expand on the conclusions in the previous review by Ouda, Profant, and Syka. The review by Williamson and Frisina reveals that temporal deficits in sound processing are one of the main physiological biomarkers that may have the potential to allow earlier precise diagnostic of age-related hearing loss needed for early preclinical recognition and clinical transfer.

23. The ability to detect the location of a sound source in space depends on central processing of both binaural and monaural acoustic information. This ability changes throughout the life span. The chapter entitled "Age-related changes in sound localization ability" by Freigang et al. (2015) presents recent advances in this field by discussing electrophysiological and behavioral data. In this article, the term 'age-related' does not simply refer to the elderly but expands to children as well as young and older adults. Perception of the acoustic space develops from an initially imprecise representation in young children (6-9 years) to a concise representation of spatial positions in young adults. The performance declines again in older adults (above 50-60 years of age). Localization accuracy is characterized by a strong deterioration in older adults, presumably due to declined processing of binaural temporal as well as monaural spectro-temporal cues. Nevertheless, a significant number of old and very old people still have low hearing thresholds and perform in discrimination tests like young adults. What causes their 'immunity' to age-related deterioration is at present unclear.

24. The final chapter by Furness (2015) explores the "Molecular basis of hair cell loss", a well-deserving theme for the final chapter in this special issue. The chapter reviews the rich set of data on various molecular and other means that ultimately result in the demise of hair cells, mostly in the form of an age-related decline of viability and eventual loss. The chapter also critically reviews several approaches that have been developed, in particular approaches to combat the formation of radicals that may eventually lead to clinical treatments to prevent or at least delay hearing loss.

The editors of this special issue want to express their gratitude to all the authors who have contributed to this special volume by providing their articles in a timely fashion and by responding positively to critical reviews in revising their papers. Special thanks are also due to Klaus Unsicker, the editorin-chief, who encouraged and supported us in putting together this special issue as invited guest editors. Our very special thanks go to Maite Menés for her editorial assistance, invaluable help with invitations to authors and reviewers, and reminding us about deadlines. Without this constant help and these reminders, this special issue would not have been possible. We like to conclude with our hopes that others in the auditory field will find the information provided in this special issue of use for their own research or for thinking about aspects of the auditory field that appear only distantly related to their own research area.

\section{References}

Davis RL, Crozier RA (2015) Dynamic firing properties of type I spiral ganglion neurons. Cell Tissue Res. doi:10.1007/ s00441-014-2071-x

Duque D, Ayala YA, Malmierca MS (2015) Deviance detection in auditory subcortical structures: what can we learn from neurochemistry and neural connectivity? Cell Tissue Res. doi:10.1007/s00441-0152134-7

Durruthy-Durruthy R, Heller S (2014) Applications for single cell trajectory analysis in inner ear development and regeneration. Cell Tissue Res. doi:10.1007/s00441-014-2079-2

Eggermont JJ, Roberts LE (2014) Tinnitus: animal models and findings in humans. Cell Tissue Res. doi:10.1007/s00441-014-1992-8

Freigang C, Richter N, Rübsamen R, Ludwig AA (2015) Age-related changes in sound localization ability. Cell Tissue Res. doi:10. 1007/s00441-015-2230-8

Friauf E, Fischer AU, Fuhr MF (2015) Synaptic plasticity in the auditory system: a review. Cell Tissue Res. doi:10.1007/s00441-015-2176-X 
Fritzsch B, Pan N, Jahan I, Elliott KL (2014) Inner ear development: building a spiral ganglion and an organ of Corti out of unspecified ectoderm. Cell Tissue Res. doi:10.1007/s00441-014-2031-5

Furness DN (2015) Molecular basis of hair cell loss. Cell Tissue Res. doi: 10.1007/s00441-015-2113-Z

Heil P, Peterson AJ (2015) Basic response properties of auditory nerve fibers: a review. Cell Tissue Res. doi:10.1007/s00441-015-2177-9

Hubka P, Konerding W, Kral A (2014) Auditory feedback modulates development of kitten vocalizations. Cell Tissue Res. doi:10.1007/ s00441-014-2059-6

King AJ (2015) Crossmodal plasticity and hearing capabilities following blindness. Cell Tissue Res. doi:10.1007/s00441-015-2175-y

Knipper M, Panford-Walsh R, Singer W, Ruttiger L, Zimmermann U (2015) Specific synaptopathies diversify brain responses and hearing disorders: you lose the gain from early life. Cell Tissue Res. doi: 10.1007/s00441-015-2168-x

Nothwang HG, Ebbers L, Schluter T, Willaredt MA (2015) The emerging framework of mammalian auditory hindbrain development. Cell Tissue Res. doi:10.1007/s00441-014-2110-7

Nouvian R, Eybalin M, Puel JL (2015) Cochlear efferents in developing adult and pathological conditions. Cell Tissue Res. doi:10.1007/ s00441-015-2158-z

Ouda L, Profant O, Syka J (2015) Age-related changes in the central auditory system. Cell Tissue Res. doi:10.1007/s00441-014-2107-2
Rudnicki M, Hemmert W (2015) Modelling auditory coding: from sound to spikes. Cell Tissue Res. doi:10.1007/s00441-015-2202-z

Ryugo D (2014) Auditory neuroplasticity, hearing loss and cochlear implants. Cell Tissue Res. doi:10.1007/s00441-014-2004-8

Seymour ML, Pereira FA (2015) Survival of auditory hair cells. Cell Tissue Res. doi:10.1007/s00441-015-2152-5

Sienknecht UJ (2015) Current concepts of hair cell differentiation and planar cell polarity in inner ear sensory organs. Cell Tissue Res. doi:10.1007/s00441-015-2200-1

Vila PM, Lieu JE (2015) Asymmetric and unilateral hearing losses in children. Cell Tissue Res. doi:10.1007/s00441-015-2208-6

Wang HC, Bergles DE (2014) Spontaneous activity in the developing auditory system. Cell Tissue Res. doi:10.1007/s00441-014-2007-5

Wichmann C, Moser T (2015) Relating structure and function of inner hair cell ribbon synapses. Cell Tissue Res. doi:10.1007/s00441-0142102-7

Williamson TT, Zhu X, Walton JP, Frisina RD (2014) Auditory brainstem gap responses start to decline in mice in middle age: a novel physiological biomarker for age-related hearing loss. Cell Tissue Res. doi:10.1007/s00441-014-2003-9

Wu C, Stefanescu RA, Martel DT, Shore SE (2014) Listening to another sense: somatosensory integration in sthe auditory system. Cell Tissue Res. doi:10.1007/s00441-014-2074-7 